\section{Reversible deafness and blindness in a patient with cryptococcal meningitis in Tanzania}

\author{
Matt Douglas-Vail,' Taylor Bechamp, ${ }^{1}$ \\ Simran Gohal, ${ }^{1}$ Robert Soegtrop, ${ }^{1}$ \\ Sarah Vitali, ${ }^{1}$ Joan Rugemalila, ${ }^{2}$ \\ Neil R.H. Stone ${ }^{3}$ \\ 'Schulich School of Medicine and \\ Dentistry, Western University, Canada; \\ 2Muhimbili National Hospital, Tanzania; \\ ${ }^{3}$ Institute for Infection and Immunity, St. \\ George's, University of London, UK
}

\section{Abstract}

Cryptococcal meningitis is a common and devastating complication of advanced HIV, and is most prevalent in low resource settings in sub Saharan Africa. Raised intracranial pressure is one of the hallmarks of the disease, which can lead to visual and hearing loss and ultimately death. We present the case of a patient with visual and hearing impairment secondary to Cryptococcal meningitis successfully managed by serial cerebrospinal fluid drainage. This case highlights some of the challenges of managing this severe opportunistic infection in a low resource setting.

\section{Introduction}

Cryptococcal meningitis (CM) is a common and severe opportunistic infection of advanced human immunodeficiency virus (HIV) infection. It is estimated to cause up to 600,000 deaths per year, ${ }^{1}$ primarily in sub Saharan Africa, and is closely linked to the HIV pandemic. CM is always fatal if left untreated, and even with access to antifungal therapy, mortality is up to $40 \%$ within 10 weeks. ${ }^{2}$ In the age of widespread access to antiretroviral therapy for HIV, surviving CM and subsequently treating the underlying HIV infection can lead to good longterm outcomes. However, treatment of this opportunistic neurological infection is challenging. Prompt antifungal therapy is central to successful treatment however management of raised intracranial pressure is an essential adjunct to therapy. Morbidity and mortality in $\mathrm{CM}$ is often related to severely elevated intracranial pressure, which can lead to visual loss, hearing loss and ultimately death. ${ }^{3}$ Moreover, the recommended antifungal treatments are often not available in parts of the world most affected by $\mathrm{CM},{ }^{4}$ and management of this complex infection presents particular challenges in resource limited settings. ${ }^{5}$ Here we describe a patient with severe, advanced $\mathrm{CM}$ in a resource-limited setting, which was effectively treated by serial lumbar punctures and intracranial pressure (ICP) control.

\section{Case Report}

A 19-year-old female was referred from a remote rural district to a tertiary referral hospital in Tanzania. She had a 3-month history of severe headache, unrelieved by analgesia with deterioration in vision over the previous 6 weeks and progressive bilateral hearing loss. The headache was associated with regular bouts of vomiting. She was known to be HIV positive and had been on antiretroviral therapy for 7 years (Tenofovir, Lamivudine, Efavirenz).

On admission she had a Glasgow Coma Score (GCS) of 15, was afebrile and normotensive. She had no neck stiffness. She was however profoundly blind with no light or dark perception in either eye. She had a marked left sided ptosis with the pupil down and out, suggestive of a $3^{\text {rd }}$ cranial nerve palsy. Fundoscopy revealed marked papilloedema bilaterally. She had severe bilateral hearing impairment, able only to hear loud voices at very close proximity. She had normal power, tone and reflexes in all limbs. CD4 count was 100 cells $/ \mathrm{mm}^{3}$. Serum creatinine $(62 \mu \mathrm{mol} / \mathrm{L})$ and ALT $(8 \mathrm{U} / \mathrm{L})$ were within normal limits.

Brain imaging not available (the site possessed a CT scanner however it was not functioning at the time due to technical issues) therefore a lumbar puncture was performed despite the papilloedema. Opening pressure (OP) was high at $37 \mathrm{~cm} \mathrm{H}_{2} 0$. The $\mathrm{CSF}$ was clear in appearance. The patient reported almost instantaneous relief of headache following the lumbar puncture. CSF analysis was positive for Cryptococcal antigen (lateral flow assay) and encapsulated yeast were seen on Indian Ink staining (Figure 1A). Gram stain was negative. CSF culture was positive for Cryptococcus neoformans on YPD agar (Figure 1B). The patient was initiated on fluconazole monotherapy at a dose of $1200 \mathrm{mg}$ per day due to the unavailability of Amphotericin B. Therapeutic lumbar punctures were performed on days $1,4,7,14$, 18 and 22 , with corresponding opening pressures of $37,37,27,31,27$ and finally $20 \mathrm{~cm}$ $\mathrm{H}_{2}$ 0. On each occasion CSF was drained to a closing pressure of $<20 \mathrm{~cm}$. Approximately 10 $\mathrm{mL}$ of CSF was removed at each LP. On day 22 the $\mathrm{OP}$ was normal at $20 \mathrm{~cm} \mathrm{H}_{2} \mathrm{O}$. On discharge her ptosis had resolved, her vision was improving to the point where she could discern light and dark and shapes. Her hearing had recovered to almost normal. She received $1200 \mathrm{mg}$ daily of oral fluconazole for the first two weeks,
Correspondence: Neil R.H. Stone, Wellcome Trust Clinical Research Fellow, Institute for Infection and Immunity, St. George's, University of London, Cranmer Terrace, London SW17 0RE, UK. Tel.: +44.208.725.2676 - Fax: +44.208.725.3487. E-mail: nstone@sgul.ac.uk

Key words: HIV; Cryptococcal meningitis; mycology; opportunistic infections; fluconazole.

Acknowledgements: NS is supported by a Wellcome Trust Strategic Award in Medical Mycology and Fungal Immunology.

Contributions: All authors contributed extensively to the case presented in this paper. NS and MDV primarily wrote the manuscript for publication.

Conflict of interest: the authors declare no potential conflict of interest.

Received for publication: 31 August 2015.

Revision received: 16 October 2015.

Accepted for publication: 17 October 2015.

This work is licensed under a Creative Commons Attribution NonCommercial 3.0 License (CC BYNC 3.0).

(C) Copyright M. Douglas-Vail et al., 2015

Licensee PAGEPress, Italy

Infectious Disease Reports 2015; 7:6173

doi:10.4081/idr.2015.6173

which was then reduced to $400 \mathrm{mg}$ once daily to be continued for 8 weeks, with follow up planned at her local HIV treatment clinic.

\section{Discussion}

This case highlights the severe complications of CM, and also several important issues in the management of $\mathrm{CM}$ in the developing world, where the vast majority of CM cases are found. The treatment of choice is a combination of Amphotericin B and flucytosine, ${ }^{6}$ neither of which are readily available in low resource settings, resulting in the use of suboptimal therapies such as fluconazole monotherapy as in the presented case. ${ }^{4}$ Additionally toxicity management is a challenge. Even where Amphotericin B can be obtained, facilities for monitoring electrolytes and renal function are often not available making safe administration problematic. ${ }^{7}$ In the presented case, electrolyte measurement was available but regular monitoring was not feasible due to financial constraints.

Raised intracranial pressure is thought to result from decreased reabsorption of CSF mechanically by the Cryptococcal organism, 
specifically the capsule of the organism blocks the arachnoid villi. ${ }^{8}$ Raised ICP is a major cause of morbidity and mortality in $\mathrm{CM}$ and can cause a spectrum of symptoms from severe headaches (the commonest presenting symptom in $\mathrm{CM}$ ), vomiting, to cranial nerve palsies and blindness and hearing loss as with our patient. Aggressive management of raised ICP by CSF drainage is a vital component in the management of $\mathrm{CM}$, and can be accurately measured by the use of a manometer. The use of manometers are recommended in international treatment guidelines, ${ }^{6,9}$ however are usually not available in resource poor setting. ${ }^{10}$ Manometers were available in this case, however in the absence of manometers the use of IV tubing as a substitute to measure ICP has been successfully used in a similar low resource setting. ${ }^{11}$

Sixth cranial nerve palsy is the commonest focal neurological deficit encountered in $\mathrm{CM}$, in which it is often a false localizing sign secondary to raised ICP, however multiple cranial nerves can be affected and $3^{\text {rd }}$ nerve paresis has been reported in the setting of severely raised ICP. ${ }^{12}$ Visual loss in CM can present as acute, catastrophic visual loss, thought to be secondary to optic nerve infiltration by the Cryptococcus organism, or a sub-acute process secondary to raised intracranial pressure. ${ }^{13}$ Visual loss can be irreversible in up to $50 \%$ of cases. $^{14}$

Hearing loss can be caused by either infiltration, ${ }^{15}$ or compression of the vestibulocochlear nerve. In this case blindness and deafness were at least partially reversed with repeated CSF drainage in combination with antifungal therapy, albeit suboptimal therapy given the inaccessibility of antifungals other than fluconazole. Reversibility of both the hearing and visual loss is highly suggestive that they were caused at least in part by the severe elevation in ICP.

The lack of access to brain imaging is also a major problem in resource-limited settings. CT imaging of the brain is recommended when and where available, particularly to exclude intracranial mass lesions. However, if the clinical index of suspicion for CM is high in a low resource setting without access to imaging, the balance of risk often favors performing a lumbar puncture. Raised intracranial pressure in this context is not a specific contraindication to LP, and this is reflected in international CM management guidelines. ${ }^{9}$ The risk of coning or herniation is reduced in $\mathrm{CM}$ as the raised ICP generally leads to a communicating rather than non-communicating hydrocephalus (which usually results from CSF flow obstruction), and multiple, high volume drainage of CSF in the specific setting of CM is considered not only safe but an essential component of management. ${ }^{16}$

\section{Conclusions}

This case demonstrates that even in severe and advanced cases of $\mathrm{CM}$, aggressive management of raised ICP can significantly improve outcomes and reduce morbidity and mortality. This can be performed by the relatively straightforward and low-tech procedure of the lumbar puncture. There is a strong body of evidence that therapeutic LPs improve out- comes in $\mathrm{CM}$, and there is growing evidence that therapeutic lumbar punctures are beneficial in the management of Cryptococcal meningitis even in the absence of raised intracranial pressure and can help to remove cryptococcal antigen. ${ }^{17,18}$

The case also highlights the particular challenges of treating $\mathrm{CM}$ in resource-limited settings, which is where the vast majority of cases worldwide are encountered. Successful treatment of $\mathrm{CM}$ relies on access to effective, rapid-

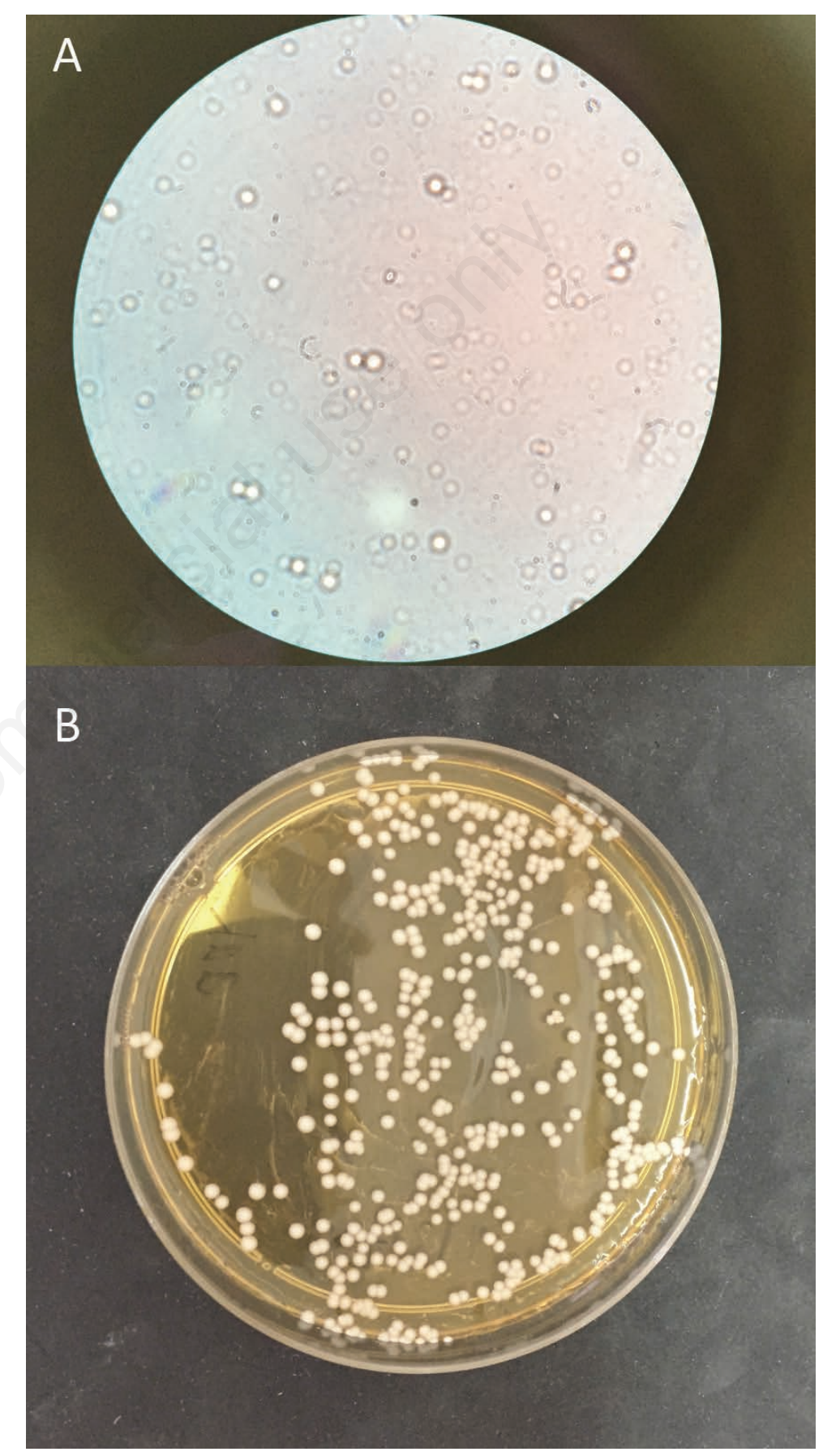

Figure 1. A) Indian Ink staining of cerebrospinal fluid revealing encapsulated yeasts; B) growth of cream colonies from cerebrospinal fluid on YPD agar. 
ly fungicidal drugs in combination with ICP control by LP followed by immune restoration with antiretroviral therapy (ART). There have been giant strides in ART rollout across Africa in recent years, leading to the prospect of longterm survival if patients can survive CM, provided ART is not commenced too early given the risk of IRIS. ${ }^{19}$ However, access to manometers remains a significant problem as accurately measuring and managing pressure is crucial, although solutions such as substituting manometers with IV tubing may be feasible. Additionally, fluconazole monotherapy remains the only option in much of the African continent, despite its inferiority to Amphotericin B based regimens.

Our case shows how effective simply performing serial LPs for patients even with the most drastic complications of CM can be, yet also demonstrates the need to campaign for greater access to both manometers and antifungal drugs in these settings.

\section{References}

1. Park BJ, Wannemuehler KA, Marston BJ, et al. Estimation of the current global burden of cryptococcal meningitis among persons living with HIV/AIDS. AIDS 2009;23:525-30.

2. Stone NR, Bicanic T. Therapy of AIDSrelated cryptococcal meningitis. Curr Treat Opt Infect Dis 2014;6:294-308.

3. de Vedia L, Arechavala A, Calderón MI, et al. Relevance of intracranial hypertension control in the management of Cryptococcus neoformans meningitis related to AIDS. Infection 2013;41:1073-7.

4. Loyse A, Dromer F, Day J, et al. Flucytosine and cryptococcosis: time to urgently address the worldwide accessibility of a 50-year-old antifungal. J Antimicrob Chemother 2013;68:2435-44.

5. Musubire AK, Meya BD, Mayanja-Kizza H, et al. Challenges in diagnosis and management of Cryptococcal immune reconstitution inflammatory syndrome (IRIS) in resource limited settings. Afr Health Sci 2012;12:226-30.

6. Perfect JR, Dismukes WE, Dromer F, et al. Clinical practice guidelines for the management of cryptococcal disease: 2010 update by the infectious diseases society of america. Clin Infect Dis 2010;50:291322.

7. Bahr NC, Rolfes MA, Musubire A, et al. Standardized electrolyte supplementation and fluid management improves survival during amphotericin therapy for cryptococcal meningitis in resource-limited settings. Open Forum Infect Dis 2014;1:ofu070.

8. Denning DW, Armstrong RW, Lewis BH, Stevens DA. Elevated cerebrospinal fluid pressures in patients with cryptococcal meningitis and acquired immunodeficiency syndrome. Am J Med 1991;91:267-72.

9. World Health Organization. Rapid advice: diagnosis, prevention and management of cryptococcal disease in HIV-infected adults, adolescents and children. December 2011. Available from: http://www.who.int/hiv/pub/cryptococcal_d isease2011/en/

10. Bicanic T, Brouwer AE, Meintjes G, et al. Relationship of cerebrospinal fluid pressure, fungal burden and outcome in patients with cryptococcal meningitis undergoing serial lumbar punctures. AIDS 2009;23:701-6.

11. Meda J, Kalluvya S, Downs JA, et al. Cryptococcal meningitis management in
Tanzania with strict schedule of serial lumber punctures using intravenous tubing sets: an operational research study. J Acquir Immune Defic Syndr 2014;66:e31-6.

12. Keane JR. Intermittent third nerve palsy with cryptococcal meningitis. J Clin Neuroophthalmol 1993;13:124-6.

13. Rex JH, Larsen RA, Dismukes WE, et al. Catastrophic visual loss due to Cryptococcus neoformans meningitis. Medicine (Baltimore) 1993;72:207-24.

14. Duggan J, Walls HM. Ocular complications of cryptococcal meningitis in patients with HIV: report of two cases and review of the literature. J Int Assoc Physicians AIDS Care (Chic) 2012;11:283-8.

15. Harada T, Sando I, Myers EN. Temporal bone histopathology in deafness due to cryptococcal meningitis. Ann Otol Rhinol Laryngol 1979;88:630-6.

16. Sun HY, Hung CC, Chang SC. Management of cryptococcal meningitis with extremely high intracranial pressure in HIV-infected patients. Clin Infect Dis 2004;38:1790-2.

17. Rolfes MA, Hullsiek KH, Rhein J, et al. The effect of therapeutic lumbar punctures on acute mortality from cryptococcal meningitis. Clin Infect Dis. 2014;59:1607-14.

18. Wijewardana I, Jarvis JN, Meintjes G, et al. Large volume lumbar punctures in cryptococcal meningitis clear cryptococcal antigen as well as lowering pressure. J Infect 2011;63:484-6.

19. Boulware DR, Meya DB, Muzoora C, et al. Timing of antiretroviral therapy after diagnosis of cryptococcal meningitis. $\mathrm{N}$ Engl $\mathrm{J}$ Med 2014;370:2487-98. 